\title{
Predictive Value of Different Parameters for Estimating the First 90-days and Long-term Survival Following Radical Cystectomy
}

\author{
(1) İsmail Selvi ${ }^{1}$, (1) Halil Başar² \\ ${ }^{1}$ Karabük University Training and Research Hospital, Clinic of Urology, Karabük, Turkey \\ 2 University of Health Sciences, Dr. Abdurrahman Yurtaslan Ankara Oncology Training and Research Hospital, Clinic of Urology, Ankara, Turkey
}

\begin{abstract}
Objective: We aimed to define the prognostic risk factors which may have an impact on the survival by assessing the effects of the different clinical parameters and several comorbidity classifications on the oncologic outcomes within the first 90 days and long-term follow-up after radical cystectomy. Materials and Methods: Fifty-two patients who underwent radical cystectomy between June 2013-and June 2017 due to bladder tumors and whose data were fully accessible, have been assessed retrospectively. The demographical, clinical and pathologic data of the patients were recorded along with the progression and mortality rates within the first 90 days follow up period and the subsequent long-term follow-up. Age-adjusted Charlson Comorbidity index $(\mathrm{ACCl})$, Eastern Cooperative Oncology Group (ECOG) score, American Society of Anesthesiologists (ASA) score, Framingham risk score, Preoperative Score to Predict Postoperative Mortality, Rockwood frailty index, preoperative serum hemogram parameters and estimated glomerular filtration rate (eGFR) were all calculated and recorded.

Results: Out of the 52 patients the average age was $68.21 \pm 6.47$, distant metastasis was observed in 17 (32.7\%) patients during monthly follow ups at an average of $37.52 \pm 26.15$ [minimum $(\min )=1$, maximum $(\max )=96$ ], while morbidity was observed in 23 patients (44.2\%). According to receiver operativing characteristic analysis, the two parameters as the most reliable tool in the prediction of the mortality during long term follow up were eGFR [area under the curve $(A \cup C)=0.754, p<0.001$ ] and Framingham score $(A \cup C=0.782, p=0.001)$. It has also been observed in multivariate analysis that Framingham score and Clavien-Dindo classification was the most meaningful predictive factor in the estimation of the mortality in the first 90 days period, progression free survival (PFS) and overall survival (OS); eGFR for PFS and OS; ECOG score for PFS; ASA score for OS; monazite/lymphocyte rate for the estimation of the mortality in the first 90 days period. In addition, pT3-4 stage has been observed to be much more meaningful in the prediction of PFS, non-transitional cell carcinoma pathology for PFS and OS, and, lymph node positivity for OS.

Conclusion: Radical cystectomy is a surgical procedure with high morbidity and mortality due to perioperative complications both in the first 90 -days of the postoperative period and long-term follow-up.We found that Framingham score and eGFR were superior and easily applicable parameters in prediction of PFS and OS, whereas Framingham score and MLR were better in prediction of the first 90-days mortality. In this way, we think that we can identify patients who are more suitable for cystectomy, so we can provide more successful postoperative follow-up and treatment management. Keywords: eGFR, Framingham risk score, monocyte/lymphocyte ratio, oncologic outcomes, radical cystectomy
\end{abstract}

\section{Introduction}

Out of the cancers, the bladder cancer is the sixth malignity which is encountered widely while it is ranked to be the second frequency out of the urologic tumors (1). It is the fifth cancer type which gives way to death in all around the world (2). By the time of diagnosis, $70 \%$ of the bladder cancer is supposed to be at the non-invasive stage while the remaining $30 \%$ is at the invasive and metastatic stage (3). The diagnosis for muscle invasive cancer could be made through the transurethral

Cite this article as: Selvi I, Başar H. The Role of Different Parameters to Predict High Risk Patients for 90-day and Long-term Morbidity and Mortality After Radical Cystectomy. Bull Urooncol 2020;19(1):20-30 
resection (TUR) and the proposed treatment for stage T2-T4a $\mathrm{N} 0-\mathrm{Nx} \mathrm{MO}$ is the radical cystectomy subsequent to clinical evaluation (3). In addition, as far as the non-muscle invasive bladder cancers (NMIBC) are concerned, radical cystectomy is one of the options for the treatment of the cases with resistance against intravesical treatments or the presence of low-grade diffusive tumors which cannot be resected completely (3).

Since the median age of the patients during diagnosis is 70 , the rate of postoperative complications reaches to $60 \%$ due to the high morbidity rates of the surgical operation with the effects of the elder age and the accompanying diseases (4). The mortality rates observed within the first 90 days of the postoperative period vary between $2.6 \%$ to $7.9 \%$. (4). The preoperative assessment of the short and long-term mortality for the geriatric population ( $>70$ age) is of particular importance in this respect (5). Hence, the patients and their families will be informed of the potential risks and the approaches to protect the bladder will perhaps be brought forward for the patients with higher morbidity. However, we come to an understanding that there exists no consensus for the nomogram or scoring system which may be the best for comorbidity of such patients, if we have a look at the literature (6).

In this study, we aimed to define the prognostic risk factors which may have an impact on the survivals taking into account the effects of the various clinical parameters and different comorbidity classifications on the oncologic assessments to be made within the first 90 days follow period subsequent to cystectomy and the long term follow period as well.

\section{Materials and Methods}

The data of 65 elder people who underwent radical cystectomy was applied by a primary surgeon due to a bladder tumor between June 2013 and June 2017 at our clinic were assessed retrospectively. Nine of the patients on whom no extended lymph node dissection application was performed and four patients data of whom were inaccessible were excluded from the study. Radical cystectomy for curative purposes, bilateral lymph node dissection and ileal conduit as urinary diversion were applied on 52 patients included in this study.

The demographical data of the patients involved, body mass index (BMI), preoperative hydronephrosis status, TUR pathology, cystectomy pathology, atypical variant histology were all recorded. Prior to cystectomy, the estimated glomerular filtration rate (eGFR) was calculated and kept under the records. For this particular calculation, Modification of Diet in Renal Disease formula which includes the parameters e.g preoperative creatinin, age, gender, and race, was used. Based on the patients' data obtained during the preoperative period, Age - adjusted Charlson comorbidity index $(\mathrm{ACCl})$, Eastern Cooperative Oncology Group (ECOG) performance score, ECOG score, Framingham risk score, Preoperative Score to Predict Postoperative Mortality (POSPOM) and Rockwood frailty index (RFI) were calculated. The serum hemogram parameters [(neutrophile/lymphocyte rate (NLR), platelet/lymphocyte rate (PLR), monocyte/lymphocyte rate (MLR), average platelet volume (MPV), red cell distribution width (RDW)] taken one week before the cystectomy were calculated individually and recorded.
The follow-up period of the patients subsequent to cystectomy, postoperative complications according to Clavien-Dindo classification, progression and mortality rates were all recorded.

\section{Age-adjusted Charlson Comorbidity Index}

This scoring is used to predict the 30 days mortality in traumatic cases or the diseases which require emergency radical surgery (7). Scoring is based on the severity of the comorbidity (Imild, moderate or severe) in the case of cardiovascular, pulmonary, gastrointestinal, urologic, neurologic and hematologic diseases. Out of 19 parameters as a total, for each parameter score between 1 to 6 is assigned to attain to the total score. For each case above 50 years of age, one more point is added against 10 years increase.

\section{Eastern Cooperative Oncology Group Performance Score}

Eastern Cooperative Oncology Group performance score is scaled between 0 and 5 to assess the general performance status of the oncology patients where zero point shows good performance and five points shows death (8).

\section{American Society of Anesthesiologists Score}

American Society of Anesthesiologists (ASA) score is the primordial scoring system between 1 and 4 based on the perioperative risks based on the physical conditions of the patients during preoperative period as defined by the ASA in 1940 (9).

\section{Framingham Risk Score}

It is a nomogram that calculates the ten-year risk of cardiovascular disease development and associated mortality. The parameters used in this scoring systems are age, gender, blood pressure value, total cholesterol, high density lipoprotein (HDL) level smoking and diabetes. Scores are assigned against each of such parameters and the total score is thus calculated. The probability of the occurrence of any cardiovascular disease for the ten years period can be found out for each of the gender in pursuance with the percentile corresponding to the scores obtained. Accordingly, $<10 \%$ shows low-risk, $10-19 \%$ intermediate risk, and $\geq 20 \%$ high risk (10).

\section{Preoperative Score to Predict Postoperative Mortality}

It is the risk score which not only predicts the potentiality of the mortality at the hospital for the patents who are to undergo surgical operation but also makes risk assessment in order to reach to a clinical solution. In this scoring system, a total of seventeen predictive factors which include age, cardiovascular, cerebra-vascular, renal, urologic, endocrine, and oncologic pathologies are defined. In case the total score assigned for each of such factors is more than 28 , it then shows the poor prognosis (7).

\section{Rockwood Frailty Index}

Frailty can be characterized through external factors which may lead to physical stress. By the time the fragile individuals within the geriatric population are followed up at the hospital, 
the risks for drops, delirium and disability increase. RFI is the nomogram used to predict the duration of hospitalization and the development of the major complications for the postoperative patients (11). Scores between 1 and 4 mean to be "no fragility", 5 and 6 "mild to moderate degree", and 7 and 9 "severe fragility"

\section{Statistical Analysis}

After evaluating the normality status with the KolmogorovSmirnov and Shapiro-Wilk tests, while identifying the characteristics of all patients, the continuous variables with normal distribution were shown as mean \pm standard deviation, the non-normally distributed variables were shown as median (25th percentile-75th percentile), and categorical variables were shown as number (percent). To define the estimated value used to predict the mortality during long-term follow-up period subsequent to radical cystectomy, receiver operating characteristic curve analysis were made. Kaplan-Meier method was used for the survival analysis and the differences amongst the patient sub groups were defined through log rank test. Cox Regression analysis was used to define the variables which may have an impact on the mortality, progression free survival (PFS) and overall survival (OS) in the first 90 days. The analyses were made through IBM SPSS Statistics 21 (IBM, Armonk, NY USA) software. $p<0.05$ value is considered statistically significant.

\section{Results}

The average age of the patients included in our study is $68.21 \pm 6.47$ ( $\min =50, \max =78$ ) while 48 patients $(92.3 \%)$ were male and four patients (7.7\%) were female. During the 37.52 \pm 26.15 ( $\min =1, \max =96$ ) months follow-up period, distant metastasis was observed on 17 patients $(2.7 \%)$ with the death of 23 (44.2\%) patients. Distant metastasis has been defined in the lymph nodes of four patients, the lungs of eight patients, bones of two patients and the liver of three patients. The demographical, clinical, pathological, and oncologic outcomes for the patients are shown in Table 1.

Table 2 shows the estimated values of the parameters to be used in the prediction of the mortality in the long-term subsequent to cystectomy. Out of such values, the eGFR [area under the curve $(A U C)=0.754, p<0.001]$ and Framingham score ( $A U C=0.782, p=0.001$ ) have been observed to be the most reliable two parameters. According to the Framingham score which makes an assessment on the risk of development of cardiovascular disease over the ten years if we classify the patients in the manner that $(<10 \%)$ is low risk, $(10-19 \%)$ is medium risk, and ( $\geq 20 \%)$ is high risk, the group observed with mortality, includes high risk patients more than the other group (53.8\% vs $23.5 \%$ ) and the patients under high risk have been observed to be assigned higher mortality (61.9\%) while we have found out that much more reliable results could be attained in the prediction of the mortality if the estimated value for Framingham score is set to be 14.5. (Table 2, Figure 1-2).

Age, gender, BMI, smoking history, cystectomy pathology, atypical variant histology in cystectomy specimen, presence of carcinoma in situ, lymphovascular invasion, presence of upstaging / downstaging, surgical margin positivity, pathological
Table 1. Patients' demographical, clinical and pathological data and oncologic results

\begin{tabular}{|c|c|}
\hline Parameters & Total $(n=52)$ \\
\hline Age & $68.21 \pm 6.47$ \\
\hline Body mass index & $25.78 \pm 3.72$ \\
\hline \multicolumn{2}{|l|}{ Gender (n, \%) } \\
\hline Male & $48(92.3)$ \\
\hline Female & $4(7.7)$ \\
\hline \multicolumn{2}{|l|}{ Smoking history (n, \%) } \\
\hline Yes & $30(57.7)$ \\
\hline No & $22(42.3)$ \\
\hline \multicolumn{2}{|l|}{ TUR-BT pathology (n, \%) } \\
\hline$-\mathrm{T} 2 \mathrm{G} 3$ & $47(90.4)$ \\
\hline - Non-TCC & $5(9.6)$ \\
\hline \multicolumn{2}{|l|}{ Cystectomy pathology (n, \%) } \\
\hline -TO & $6(11.5)$ \\
\hline$-\mathrm{Ta}$ & $1(1.9)$ \\
\hline$-\mathrm{T} 2$ & $21(40.4)$ \\
\hline$-\mathrm{T3}$ & $12(23.1)$ \\
\hline$-\mathrm{T} 4$ & $7(13.5)$ \\
\hline - Non-TCC & $5(9.6)$ \\
\hline \multicolumn{2}{|l|}{ Atypical variant histology (n, \%) } \\
\hline -Micro papillary & $4(7.7)$ \\
\hline -Sarcomatoid & $10(19.2)$ \\
\hline - No atypical variant histology & $38(73.1)$ \\
\hline \multicolumn{2}{|c|}{ Upstaging after cystectomy (n, \%) } \\
\hline Yes & $23(44.2)$ \\
\hline No & $29(55.8)$ \\
\hline \multicolumn{2}{|c|}{ Downstaging after cystectomy (n, \%) } \\
\hline Yes & $7(13.5)$ \\
\hline No & $45(86.5)$ \\
\hline \multicolumn{2}{|l|}{ Accompanying CIS (N, \%) } \\
\hline Yes & $13(25.0)$ \\
\hline No & $39(75.0)$ \\
\hline \multicolumn{2}{|l|}{ Positive surgical margin (n, \%) } \\
\hline Yes & $10(19.2)$ \\
\hline No & $42(80.8)$ \\
\hline \multicolumn{2}{|l|}{ Lymph vascular invasion (n, \%) } \\
\hline Yes & $7(13.4)$ \\
\hline No & $45(86.6)$ \\
\hline \multicolumn{2}{|c|}{ Positive lymph node $(\mathrm{pN}+)(\mathrm{n}, \%)$} \\
\hline Yes & $20(38.5)$ \\
\hline No & $32(61.5)$ \\
\hline \multicolumn{2}{|c|}{ Preoperative hydronephrosis (n, \%) } \\
\hline Yes & $27(51.9)$ \\
\hline No & $25(48.1)$ \\
\hline $\operatorname{eGFR}\left(\mathrm{mL} / \mathrm{dk} / 1.73 \mathrm{~m}^{2}\right)$ & $74.84 \pm 9.92$ \\
\hline
\end{tabular}




\begin{tabular}{|c|c|}
\hline $\mathrm{ACCl}$ & $5.25 \pm 2.37$ \\
\hline \multicolumn{2}{|l|}{ Neo-adjuvant chemotherapy (n, \%) } \\
\hline Yes & $9(17.3)$ \\
\hline No & $43(82.7)$ \\
\hline \multicolumn{2}{|l|}{ Adjuvant chemotherapy (n, \%) } \\
\hline Yes & $12(23.1)$ \\
\hline No & 40 (76.9) \\
\hline ECOG score & $2(2-3)$ \\
\hline POSPOM score & $27.17 \pm 9.92$ \\
\hline Framingham score & $17.02 \pm 8.10$ \\
\hline \multicolumn{2}{|l|}{ Framingham risk classification } \\
\hline Low risk & $12(23.1)$ \\
\hline Intermediate risk & $19(36.5)$ \\
\hline High risk & $21(40.4)$ \\
\hline ASA score & $2(2-3)$ \\
\hline RFI & $5.83 \pm 1.86$ \\
\hline NLR & $2.23 \pm 0.72$ \\
\hline PLR & $113.18 \pm 28.37$ \\
\hline MLR & $0.27 \pm 0.09$ \\
\hline MPV & $3.61 \pm 0.99$ \\
\hline RDW & $13.61 \pm 0.99$ \\
\hline \multicolumn{2}{|l|}{ Clavien-Dindo classification (n, \%) } \\
\hline-1 & $22(42.3)$ \\
\hline-2 & $17(32.7)$ \\
\hline$-3 a$ & $6(11.5)$ \\
\hline$-3 b$ & $6(11.5)$ \\
\hline$-4 b$ & $1(1.9)$ \\
\hline Follow up period (month) & $37.52 \pm 26.15$ \\
\hline Rate of metastasis during follow up (n, \%) & $17(32.7)$ \\
\hline Rate of mortality in the first 90 days $(n, \%)$ & $6(11.5)$ \\
\hline General mortality rate (\%) & $23(44.2)$ \\
\hline \multicolumn{2}{|c|}{$\begin{array}{l}\text { BMI Body/Mass index, TUR-BT: Transurethral resection of the bladder tumor } \\
\text { TCC: Transitional cell carcinoma, CIS: Carcinoma in situ, eGFR: Estimatec } \\
\text { glomerular filtration rate, ACCI: Age Adjusted Charlson Comorbidity Index } \\
\text { ECOG: Eastern Cooperative Oncology Group, POSPOM: Preoperative Scor } \\
\text { to Predict Postoperative Mortality, ASA: American society of anesthesiologists } \\
\text { RFI: Rockwood frailty index, NLR: Neutrophile/lymphocyte rate, PLR: Platelet } \\
\text { lymphocyte rate, MLR: Monocyte/lymphocyte rate, MPV: Average platele } \\
\text { volume, RDW: Erythrocyte distribution width }\end{array}$} \\
\hline
\end{tabular}

lymph node positivity, preoperative hydronephrosis status, neoadjuvant/adjuvant chemotherapy, status POSPOM, Framingham score, RFI, NLR, PLR, MLR and Clavien-Dindo classification were used, univariate and multivariate survival analyzes were performed to include these data as independent variables. Since no meaningful estimated value has been defined for MPV (AUC=0.601, $p=0.214)$ and RDW $(A \cup C=0.645$, $\mathrm{p}=0.074)$ in receiver operating characteristic analysis, these two parameters have not been included in the univariate and multivariate analysis.
The predictive factors for PFS and OS within the follow-up period in the long-term, and the first 90 days mortality subsequent to radical cystectomy according to the univariate and multivariate analyses are shown in Table 3A-C. Kaplan-Meier curve for the parameters used in the prediction of the OS in accordance with the multivariate analysis is shown in Figure 1-7.

\section{Discussion}

Radical cystectomy is the surgical procedure with high morbidity and mortality both in the course of the first 90 days of the postoperative period and the follow-ups in the long-term due to the perioperative complications (7). Different publications

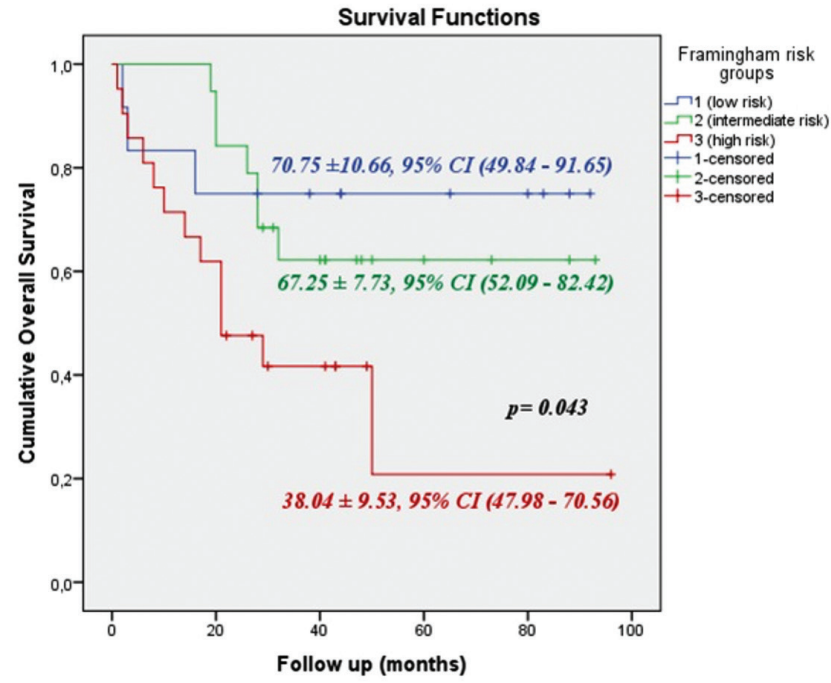

Figure 1. Kaplan-Meier plot of overall survival times predicted for Framingham risk groups

Cl: Confidence interval

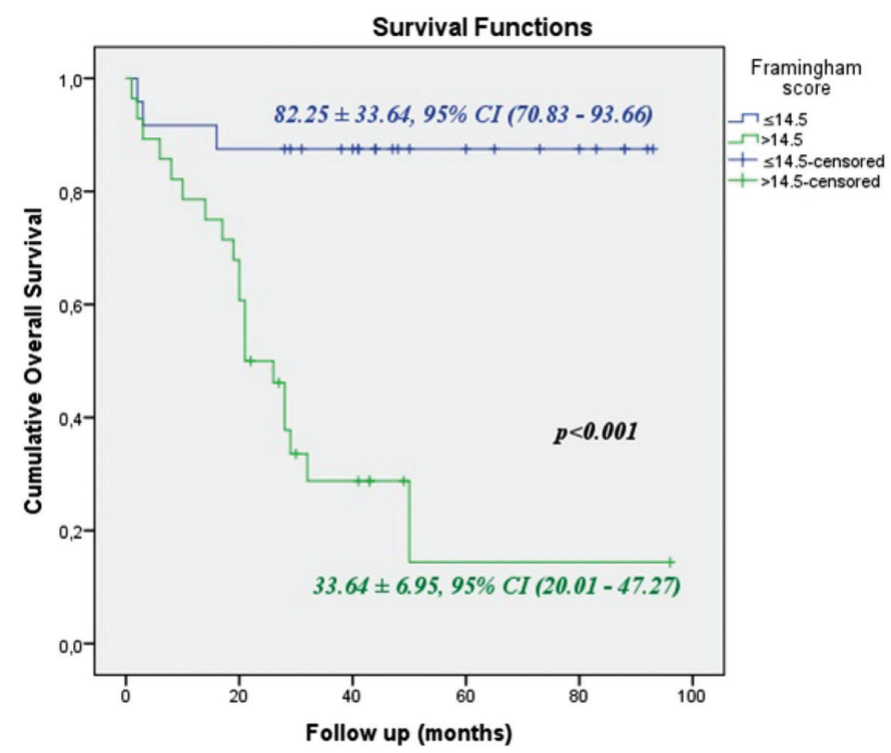

Figure 2. Kaplan-Meier plot of overall survival times predicted by Framingham risk score

$\mathrm{Cl}$ : Confidence interval 
Selvi and Başar. Prediction of Survival After Radical Cystectomy

Table 2. Cut-off values for the parameters used to predict the mortality in the long-term follow-up subsequent to radical cystectomy

\begin{tabular}{|c|c|c|c|c|c|c|c|c|}
\hline & $\mathrm{ACCl}$ & POSPOM & Framingham score & RFI & NLR & MLR & PLR & eGFR \\
\hline Cut-off value & 5.5 & 25.5 & 14.5 & 5.5 & 2.13 & 0.26 & 110.36 & 74.10 \\
\hline Sensitivity (\%) & 60.9 & 65.2 & 87.0 & 73.9 & 60.9 & 73.9 & 73.9 & 82.6 \\
\hline PPV (\%) & 66.2 & 67.2 & 75.9 & 60.4 & 57.6 & 68.1 & 68.1 & 72.7 \\
\hline NPV (\%) & 63.8 & 65.3 & 84.7 & 66.4 & 58.8 & 71.5 & 71.5 & 79.8 \\
\hline
\end{tabular}

ACCl: Age-adjusted Charlson Comorbidity index, RFI: Rockwood frailty index, POSPOM: Preoperative Score to Predict Postoperative Mortality, NLR: Neutrophile/ lymphocyte rate, MLR: Monocyte/lymphocyte rate, PLR: Platelet/lymphocyte rate, eGFR: Estimated glomerular filtration rate, PPV: Positive predictive value, NPV: Negative predictive value, AUC: Area under the curve * $\mathrm{p}<0.05$ (There is a significant difference between the groups)

have specified that the perioperative mortality ratio is $2-13 \%$ and general survival percentage for five years is $50-60 \%$ (12). We have observed that the said ratios are respectively $11.5 \%$ and $44.2 \%$ in accordance with the literature. Multimodal treatments for the protection of the bladders or radiotherapy for palliative purposes may be an option for the patients with high morbidity especially the elder patients, to get rid of the surgical risks despite the fact that such treatments are not deemed to be the alternatives to the cystectomy in view of curative treatment. Therefore, it is of particular concern to define the patients with high mortality ratio and morbidity associated with cystectomy (13).

There exists surplus of studies which pertain to the comorbidity index to be used in the prediction of the OS in the follow up in the long term and the perioperative mortality of 90 days for the patients on which radical cystectomy is planned due to bladder tumors $(4,12,13,14)$. Different studies have shown that the elder age, angina pectoris, smoking history, chronic lung diseases and Diabetes Mellitus were used as independent prognostic factors to predict the 10-year mortality subsequent to cystectomy $(13,15)$. However, instead of evaluating the comorbid conditions individually, it is thought to be more valuable to use nomograms that examine the total morbidity burden (2).

According to the updated guidelines of the European Association of Urology (EAU), it is suggested to make an evaluation for the morbidity of the potential patients who are to undergo radical cystectomy preoperatively (3). For this purpose, the widely used scoring systems are ACCI and ASA and it has been observed that they are much more beneficial for the prediction of the complications which may develop in the elder patients during the first 30 days after the operation and the cancer free mortality in the first 90 days $(13,14)$. It has been reported that the prognostic value of $\mathrm{ACCl}$ may be higher in the estimation of five-year mortality due to all causes (12).

It is obvious that the predicted values of the survival analyses of the scoring systems vary in every study $(4,12,16)$. As a matter of fact elder age $(\geq 75)$, it has been reported that progressive cancer stages (T3-4) and high ASA score ( $\geq 3)$, increase the perioperative mortality within the 90 days period (4); make a negative impact of the five-years OS ratios through $\mathrm{ACCl}$ (17) and ECOG score has no additional contribution for the prediction of the 90 days mortality (4). According to Boorjian et. al (12), ASA and ECOG scores make an impact on both the 90-days perioperative mortality and the five years OS, on the other hand $\mathrm{ACCl}$ predicts the $\mathrm{OS}$ in a much more meaningful manner. Froehner et al. (16) have specified that ACCI and ASA scores fail to predict the short and long-term mortality for the patients aged 80 and more, after cystectomy. According to the multivariate analysis in our study, we observed that advanced tumor stage (T3-4) and ECOG score affect PFS more significantly, and ASA score affects OS more significantly, while we could not determine the effect of patient age on oncological results. In addition, we have found out that non-transitional cell crcinoma pathology is much more deterministic in the prediction of the PFS and OS while positive lymph node is a determinant for the prediction of the OS. Being distinct from the publications listed in the literature we have also observed that Framingham score and Clavien-Dindo classification are the predictive factors which are much more meaningful in the determination of the first 90 days mortality, PFS and OS and eGFR is for the PFS and OS.

RFI has been in use for the estimation of the duration of hospitalization and development of major complications during postoperative period especially within the geriatric population. There exists no study showing the use of the same for the old population who underwent cystectomy (18). In our study in which we have found out that high RFI (>5.5) values decreases the OS at a rate of 2.32 in the case univariate analysis and that RFI has no meaningful impact on OS in the multi variate analysis..

Froehner et al. (5) reported that systemic disease load is effective on the survival in the long-term subsequent to cystectomy. POSPOM is one of the disease-counting classifications that can be used to predict this. In our study, although high POSPOM (> 25.5) scores decreased PFS at a rate of 2.94 times and OS at a rate of 2.37 times in univariate analysis, it did not have a significant effect in multivariate analysis.

While $28(53.8 \%)$ of 52 patients who underwent cystectomy in our study were 70 years old and under, 24 (46.2\%) were over 70 years old. Although we found that the patient's age did not affect oncological results, the rate of patients who underwent cystectomy over 75 years of age was $11.5 \%$ in accordance with the literature. Even for the cases with low ASA score $(\leq 2)$, it is observed that radical cystectomy was applicable on the patients older than 75 years old, at a lesser rate (21\% vs 49\%) (19). 
Nevertheless, Boorjian et al. (12), has stated that the elder age of the patients has no impact on the perioperative mortality and that the age is not the sole determinant for the decision to apply cystectomy In our study, 28 patients (53.8\%) aged 70 or younger, out of 52 patients received cystectomy while 24 patients (46.2) were older than 70. Although we have found out that the age of the patients had no impact on the oncologic results, the percentile of the patients who under cystectomy and aged 75 and older is $11.5 \%$ in compatible with the literature.

The updated EAU guidelines suggest the use of the Cumulative Illness Score Rating-Geriatrics (CISR-G) in the assessment of the general medical condition of the patients and when defining the most appropriate treatment alternative for the geriatric group in the case of prostate cancer (20). We consider that this scoring system may not only be used for the patients diagnosed with prostate cancer in daily practice but also for defining the geriatric patients who are to undergo cystectomy. However, the fact that this scale includes many detailed queries concerning the systemic diseases, it is neither practical nor it is possible to apply the same within the scope of the retrospective studies. Therefore, it seems much more convenient to use it in the prospective studies concerning the follow ups on the patients on whom cystectomy was applied. Froehner et al. (15) has reported that ACCl, when compared to CISR-G, is much easier to apply and is at acceptable levels to predict the postoperative mortality. Since the use of angina pectoris classification of the New York Heart Association (NYHA) and Canadian Cardiovascular Society (CCS) for the prediction of pre-cystectomy cardiac mortality

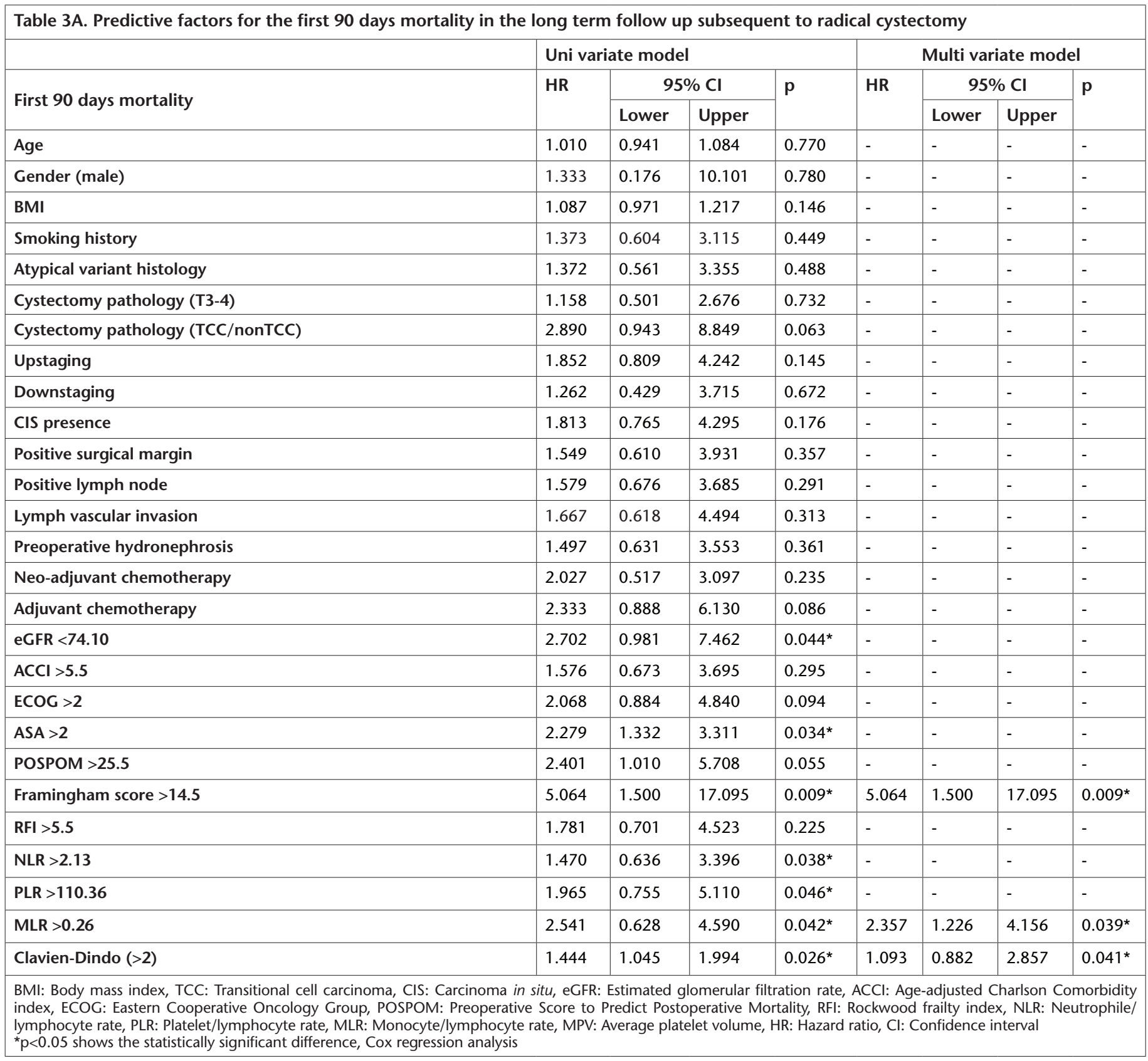




\begin{tabular}{|c|c|c|c|c|c|c|c|c|c|}
\hline \multirow{3}{*}{ Survival without progression } & \multicolumn{4}{|c|}{ Uni variate model } & \multicolumn{5}{|c|}{ Multi variate model } \\
\hline & \multirow[t]{2}{*}{ HR } & \multicolumn{2}{|c|}{$95 \% \mathrm{Cl}$} & \multirow[t]{2}{*}{ p } & \multirow[t]{2}{*}{ HR } & \multicolumn{2}{|c|}{$95 \% \mathrm{Cl}$} & \multirow{2}{*}{\multicolumn{2}{|c|}{ p }} \\
\hline & & Lower & Upper & & & Lower & Upper & & \\
\hline Age & 1.002 & 0.915 & 1.096 & 0.972 & - & - & - & \multicolumn{2}{|l|}{-} \\
\hline Gender (male) & 1.440 & 0.329 & 6.308 & 0.629 & - & - & - & \multicolumn{2}{|l|}{-} \\
\hline BMI & 1.035 & 0.913 & 1.173 & 0.590 & - & - & - & \multicolumn{2}{|l|}{-} \\
\hline Smoking history & 1.308 & 0.503 & 3.401 & 0.581 & - & - & - & \multicolumn{2}{|l|}{-} \\
\hline Atypical variant histology & 1.925 & 0.709 & 5.232 & 0.199 & - & - & - & \multicolumn{2}{|l|}{-} \\
\hline Cystectomy pathology (T3-4) & 1.569 & 0.605 & 4.070 & $0.034^{*}$ & 1.354 & 0.917 & 3.332 & \multicolumn{2}{|l|}{$0.034^{*}$} \\
\hline Cystectomy pathology (TCC/nonTCC) & 4.756 & 2.044 & 12.232 & $0.002^{*}$ & 3.756 & 1.044 & 13.222 & \multicolumn{2}{|l|}{$0.002^{*}$} \\
\hline Upstaging & 2.878 & 1.060 & 7.813 & $0.038^{*}$ & - & - & - & \multicolumn{2}{|l|}{-} \\
\hline Down staging & 2.724 & 0.761 & 7.408 & 0.331 & - & - & - & \multicolumn{2}{|l|}{-} \\
\hline CIS presence & 2.553 & 0.967 & 6.739 & 0.059 & - & - & - & \multicolumn{2}{|l|}{-} \\
\hline Positive surgical margin & 1.827 & 0.642 & 5.195 & 0.259 & - & - & - & \multicolumn{2}{|l|}{-} \\
\hline Positive lymph node & 1.924 & 0.739 & 5.015 & 0.180 & - & - & - & \multicolumn{2}{|l|}{-} \\
\hline Lymph vascular invasion & 2.649 & 0.858 & 8.176 & 0.090 & - & - & - & \multicolumn{2}{|l|}{-} \\
\hline Preoperative hydronephrosis & 2.116 & 0.781 & 5.734 & 0.141 & - & - & - & \multicolumn{2}{|l|}{-} \\
\hline Neo-adjuvant chemotherapy & 3.303 & 0.837 & 6.835 & 0.168 & - & - & - & \multicolumn{2}{|l|}{-} \\
\hline Adjuvant chemotherapy & 1.688 & 0.589 & 4.837 & 0.329 & - & - & - & \multicolumn{2}{|l|}{-} \\
\hline$e G F R<74.10$ & 4.739 & 1.540 & 14.705 & $0.007^{*}$ & 4.504 & 1.443 & 14.084 & \multicolumn{2}{|l|}{$0.002^{*}$} \\
\hline $\mathrm{ACCl}>5.5$ & 5.672 & 1.840 & 17.481 & $0.003^{*}$ & - & - & - & \multicolumn{2}{|l|}{-} \\
\hline ECOG >2 & 2.993 & 1.102 & 8.128 & $0.031^{*}$ & 3.036 & 1.003 & 9.190 & \multicolumn{2}{|l|}{$0.049^{*}$} \\
\hline ASA $>2$ & 3.419 & 1.259 & 9.283 & $0.016^{*}$ & - & - & - & - & - \\
\hline POSPOM > 25.5 & 2.941 & 1.032 & 8.385 & $0.044^{*}$ & - & - & - & - & - \\
\hline Framingham score $>\mathbf{1 4 . 5}$ & 5.845 & 1.661 & 20.573 & $0.006^{*}$ & 4.466 & 1.128 & 107.684 & $0.033^{*}$ & - \\
\hline $\mathrm{RFI}>5.5$ & 1.523 & 0.562 & 4.132 & 0.408 & - & - & - & - & - \\
\hline NLR $>2.13$ & 1.825 & 0.675 & 4.936 & $0.036^{*}$ & - & - & - & - & - \\
\hline PLR>110.36 & 2.534 & 1.172 & 8.842 & $<0.001^{*}$ & - & - & - & - & - \\
\hline MLR $>0.26$ & 2.452 & 1.843 & 6.591 & $0.004^{*}$ & - & - & - & - & - \\
\hline Clavien-Dindo (>2) & 3.409 & 1.230 & 9.451 & $0.018^{*}$ & - & 2.442 & 1.346 & 6.773 & $0.024^{*}$ \\
\hline
\end{tabular}

may vary both due to the requirement for clinical familiarity and the estimation of the mortality risk of any given patient, it seems much appropriate to make use of ASA score and ACCI in lieu of these (21). We have observed in our study that the Framingham risk scores which predict the cardiovascular mortality over a period of 10 years is much more successful in the prediction of the first 90 days mortality, PFS and OS when compared with ASA, ECOG, ACCI, RFI and POSPOM. Hence, we consider that Framingham risk score which is much more practical method of calculation in comparison with the $\mathrm{ACCl}$, NYHA and CCS angina pectoris classification, is much easier to use in the analysis to be made to predict the survival after cystectomy.

Out of the other mortality indices for survival after cystectomy in the literature are the Elixhauser index, Lee mortality index and Adult Comorbidity Evaluation-27 which all are more popular. $(4,12,15)$. However, none of such indices has superiority over
$\mathrm{ACCl}, \mathrm{ECOG}$ and ASA in view of easiness in the application and high prediction rates (4). Although we have found out that ACCI, ECOG and ASA predicted the first 90 days mortality, PFS and $O S$ in the univariate analysis in a meaningful manner, the ECOG score has predicted the PFS and ASA score has predicted the OS in a meaningful manner in the multi variate analysis.

In the literature, it has been reported that decreased preoperative eGFR values negatively affect GS in renal cell carcinoma by increasing cancer-specific survival (CSS), recurrence-free survival and cardiovascular risk $(22,23)$. Matsumoto et al. (24) has reported that there observed a distinct decrease in eGFR levels subsequent to radical cystectomy, in case the eGFR value is $<45 \mathrm{~mL} / \mathrm{min} / 1.73 \mathrm{~m}^{2}$ the PFS and CSS decreases significantly. In the multivariate analysis, we observed that preoperatively decreased eGFR levels $\left(<74.10 \mathrm{~mL} / \mathrm{min} / 1.73 \mathrm{~m}^{2}\right)$ decreased PFS by 4.73 times and OS by 4.10 times after cystectomy. In 


\begin{tabular}{|c|c|c|c|c|c|c|c|c|}
\hline \multirow{3}{*}{ General survival } & \multicolumn{4}{|c|}{ Uni variate model } & \multicolumn{4}{|c|}{ Multi variate model } \\
\hline & \multirow[t]{2}{*}{ HR } & \multicolumn{2}{|c|}{$95 \% \mathrm{Cl}$} & \multirow[t]{2}{*}{ p } & \multirow[t]{2}{*}{$\mathrm{HR}$} & \multicolumn{2}{|c|}{$95 \% \mathrm{Cl}$} & \multirow[t]{2}{*}{ p } \\
\hline & & Lower & Upper & & & Lower & Upper & \\
\hline Age & 1.064 & 0.973 & 1.164 & 0.171 & - & - & - & - \\
\hline Gender (male) & 2.074 & 0.278 & 15.384 & 0.477 & - & - & - & - \\
\hline BMI & 1.048 & 0.942 & 1.166 & 0.389 & - & - & - & - \\
\hline Smoking history & 1.655 & 0.729 & 13.759 & 0.227 & - & - & - & - \\
\hline Atypical variance & 1.408 & 0.576 & 3.440 & 0.453 & - & - & - & - \\
\hline Cystectomy pathology (T3-4) & 1.079 & 0.466 & 2.498 & $0.039^{*}$ & - & - & - & - \\
\hline Cystectomy pathology (TCC/nonTCC) & 3.802 & 1.226 & 11.764 & $0.021^{*}$ & 4.901 & 1.345 & 17.857 & $0.016^{*}$ \\
\hline Upstaging & 1.862 & 0.813 & 4.267 & $0.042^{*}$ & - & - & - & - \\
\hline Down staging & 1.463 & 0.495 & 4.328 & 0.491 & - & - & - & - \\
\hline CIS presence & 1.754 & 0.742 & 4.148 & 0.201 & - & - & - & - \\
\hline Positive surgical margin & 1.494 & 0.587 & 3.803 & 0.403 & - & - & - & - \\
\hline Positive lymph node & 2.787 & 1.212 & 6.407 & $0.016^{*}$ & 2.671 & 1.147 & 6.220 & $0.001^{*}$ \\
\hline Lymph vascular invasion & 2.727 & 1.067 & 6.971 & $0.036^{*}$ & - & - & - & - \\
\hline Preoperative hydronephrosis & 2.109 & 0.889 & 5.006 & $0.031^{*}$ & - & - & - & - \\
\hline Neo-adjuvant chemotherapy & 2.258 & 0.511 & 3.002 & 0.123 & - & - & - & - \\
\hline Adjuvant chemotherapy & 1.500 & 0.614 & 3.664 & 0.374 & - & - & - & - \\
\hline eGFR $<74.10$ & 4.102 & 1.865 & 13.888 & $0.001^{*}$ & 3.264 & 1.445 & 9.603 & $0.017^{*}$ \\
\hline $\mathrm{ACCl}>5.5$ & 2.630 & 1.131 & 6.116 & $0.025^{*}$ & - & - & - & - \\
\hline $\mathrm{ECOG}>2$ & 2.555 & 1.685 & 3.531 & $0.029^{*}$ & - & - & - & - \\
\hline ASA >2 & 2.810 & 0.796 & 4.118 & $0.007^{*}$ & 2.789 & 1.572 & 4.512 & $0.024^{*}$ \\
\hline POSPOM > 25.5 & 2.370 & 0.998 & 5.627 & $0.041^{*}$ & - & - & - & - \\
\hline Framingham score $>14.5$ & 5.973 & 2.612 & 16.825 & $<0.001^{*}$ & 5.973 & 2.612 & 16.825 & $<0.001^{*}$ \\
\hline $\mathrm{RFI}>5.5$ & 2.329 & 0.915 & 5.928 & $0.007^{*}$ & - & - & - & - \\
\hline NLR $>2.13$ & 2.407 & 0.609 & 3.252 & $0.004^{*}$ & - & - & - & - \\
\hline PLR > 110.36 & 2.631 & 1.425 & 9.253 & $0.007^{*}$ & - & - & - & - \\
\hline MLR $>0.26$ & 4.287 & 1.666 & 11.029 & $0.003^{*}$ & - & - & - & - \\
\hline Clavien-Dindo (>2) & 4.592 & 2.005 & 10.516 & $<0.001^{*}$ & 5.005 & 1.547 & 16.192 & $0.007^{*}$ \\
\hline \multicolumn{9}{|c|}{$\begin{array}{l}\text { BMI: Body mass index, TCC: Transitional cell carcinoma, CIS: Carcinoma in situ, eGFR: Estimated glomerular filtration rate, ACCI: Age-adjusted Charlson Comorbidity index } \\
\text { ECOG: Eastern Cooperative Oncology Group, POSPOM: Preoperative Score to Predict Postoperative Mortality, RFI: Rockwood frailty index, NLR: Neutrophile/lymphocyt } \\
\text { rate, PLR: Platelet/lymphocyte rate, MLR: Monocyte/lymphocyte rate, MPV: Average platelet volume, HR: Hazard ratio, Cl: Confidence interval } \\
\text { * }<<0.05 \text { shows the statistically significant difference, Cox regression analysis }\end{array}$} \\
\hline
\end{tabular}

univariate analysis, preoperative eGFR $<74.10$ values reduce the first 90 -day mortality by 2.70 times. We are in the thought that the decrease to be observed in the basal eGFR values within any longer term will have a negative impact both in view of the renal function and the cardiovascular risks as supported by such findings.

Recently, there observed an increase in the number of studies examining the effects of the serum hemogram parameters and inflammatory determinants on the oncologic results as far as the cancers of genitourinary system are concerned (25). During tumor proliferation, it is considered that there observed an increase in the rate of neutrophile due to the effects of the inflammatory cytokines primarily the interleukin -6 and the granulocyte colony stimulating factors (26). Decrease in the lymphocytes as a consequence of the reactions against the inflammatory cases and increase in the tumor related macrophages derived from the monocyte in circulation have been envisaged. The tumor related macrophages expedite the tumor progression (26). As per these assumptions, we may conclude that NLR and MLR may increase for the individuals with surplus of tumor progression.

The high NLR $(\geq 2.7)$ levels measured prior to the radical cystectomy are found to be in the relation with both the progressed pathological tumor stages and the decreased CSS and OS (27). Another study showed that the high NLR (>4.33), preoperative hydronephrosis and preoperative tumor stages are in relation with upstaging of T3 stage of the disease after the cystectomy (28). In different studies, low lymphocyte/ monocyte ratio $(<2.44)$ and high PLR $(>111)$ values have been reported to predict decreased CSS and OS after cystectomy (29). Despite all these facts, according to Schulz et al. (30), the low PLR $(\leq 28)$ values are in relation with the progressed 


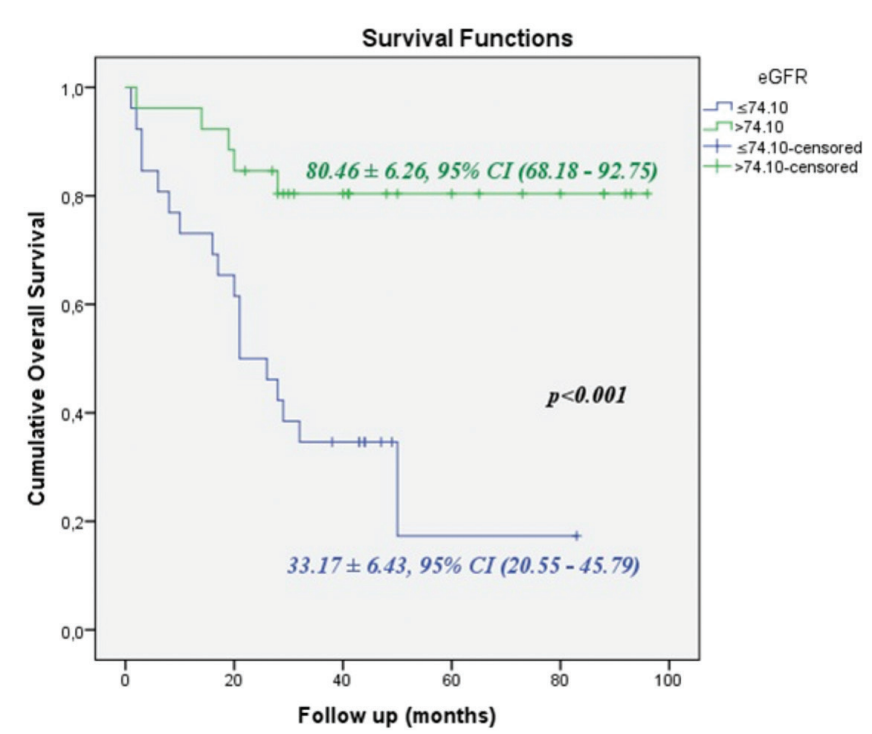

Figure 3. Graphics for Kaplan-Meier analysis showing the estimated durations of overall survival based on the eGFR values

$\mathrm{Cl}$ : Confidence interval, eGFR: predictive glomerular filtration rate

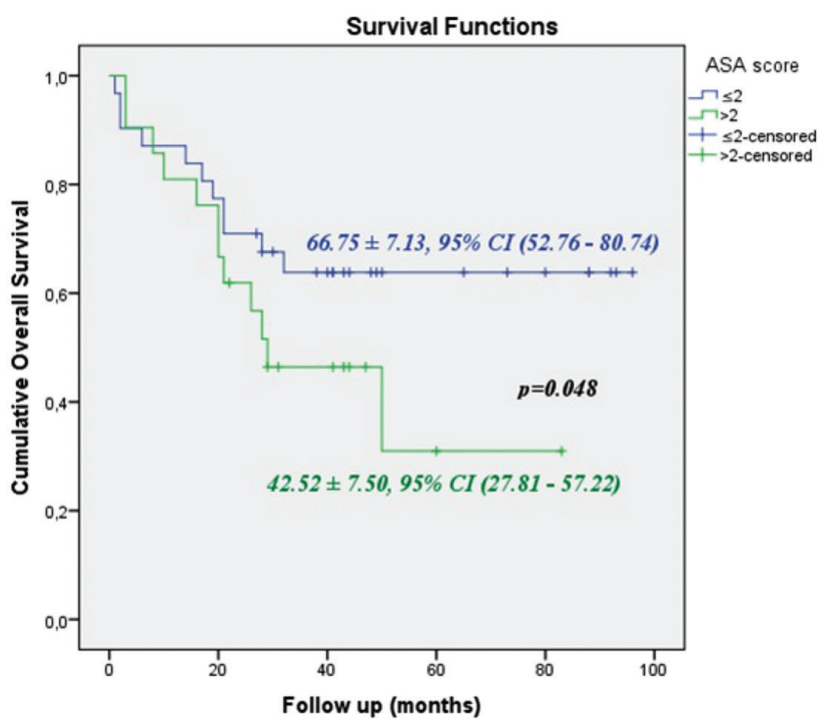

Figure 4. Graphics for Kaplan-Meier analysis showing the estimated durations of overall survival based on the ASA scores

$\mathrm{Cl}$ : Confidence interval, ASA: American Society of Anesthesiologists

tumor stages and decreased survival. Yoshida et al. (26) associated low perioperative LMR with shorter CSS and OS, suggesting that predictive power is better than NLR. Based on our findings, although the high NLR (>2.13), PLR (>110.36) and MLR (>0.26) levels predict the first 90 days mortality , PFS and OS significantly according to univariate analysis, high MLR increases the first 90 -day mortality by 2.35 times according to multivariate analysis.

\section{Study Limitations}

The patients whose data were accessible were included in the study without a statistically significant sample size and

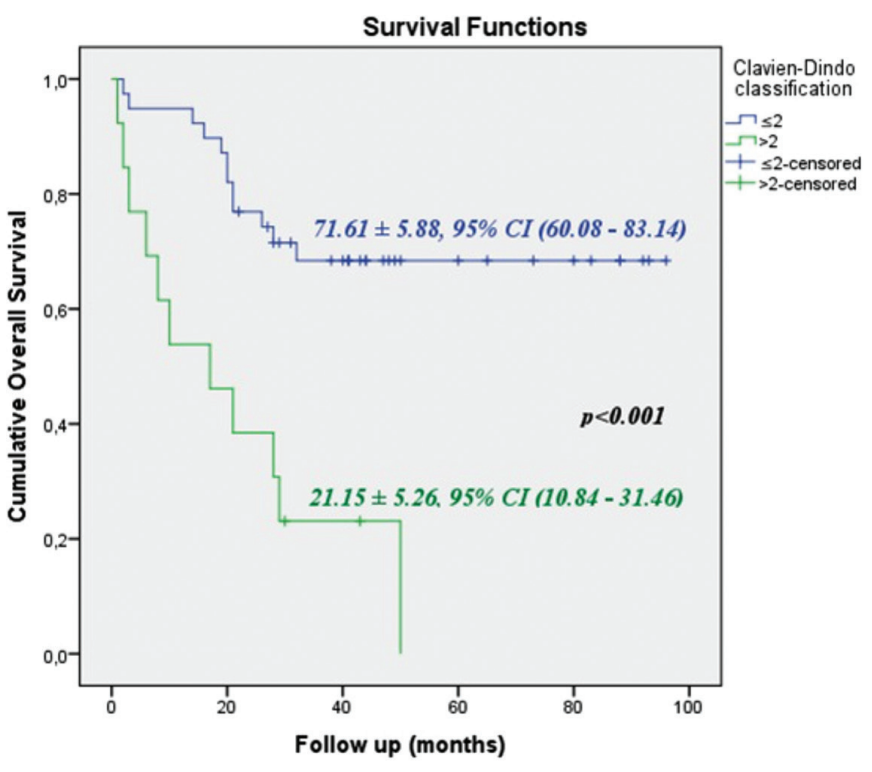

Figure 5. Graphics for Kaplan-Meier analysis showing the estimated durations of overall survival based on the Clavien-Dindo classification

$\mathrm{Cl}$ : Confidence interval

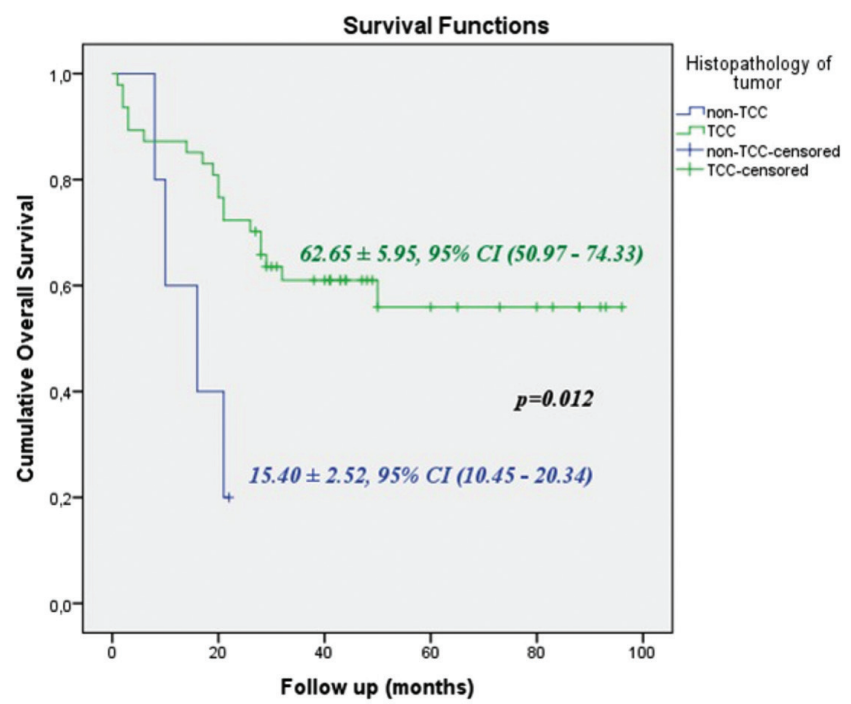

Figure 6. Graphics for Kaplan - Meier analysis showing the estimated durations of overall survival based on the tumor histology

$\mathrm{Cl}$ : Confidence interval, TCC: Transitional cell carcinoma

randomization due to the restricted number of the patients. Therefore, the major restrictive elements of our study are the retrorespective design of the study limited number of patients involved, short follow-up period and follow-up results belong to a single center without non-randomization. The patents underwent operation by the same surgeon and the same techniques to reduce the effects of the distinctive surgical experience on the outcomes attained. However, over the 4years period of the study, the same primary surgeon executed cystectomy operation at an annual average rate of 16.25 . If we consider that an annual number of 20-25 operations are executed at the high-volume centers, the low volume of cases in 


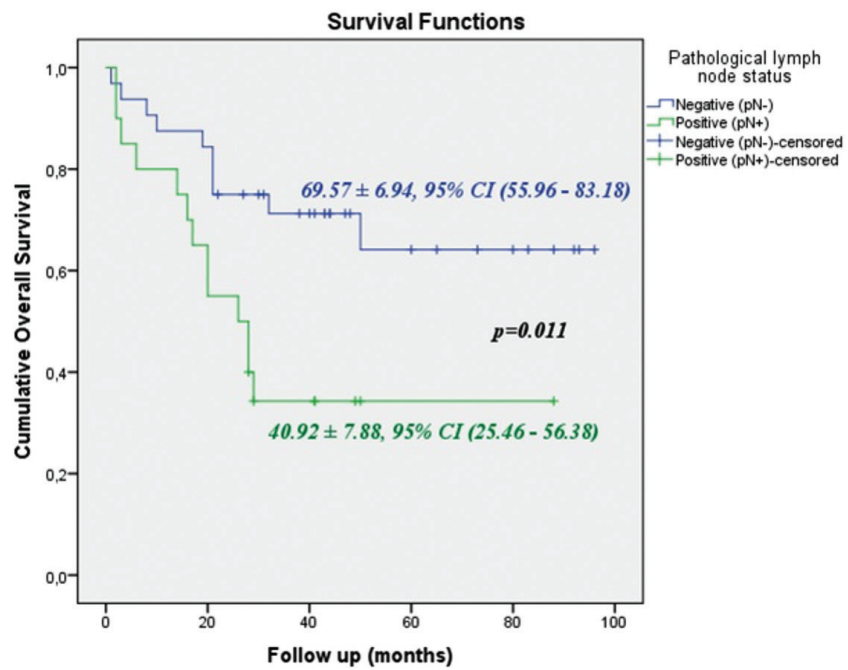

Figure 7. Graphics for Kaplan-Meier analysis showing the estimated durations of overall survival based on the status of the pathologic lymph node

our study may be deemed to be another restrictive factor which may have an impact on the results. In addition, since neoadjuvant or adjuvant chemotherapy was not applied in all cases of muscle invasion bladder cancer, we are in the thought that the heterogeneous tumor characteristics observed on the patients involved in our study might have caused a restrictive effect on the results of the survival. Nevertheless, the patients on whom no extended lymph node dissection was carried out, we have made every effort to make an assessment about the standardized group of patients as part of the surgical method applied.

\section{Conclusions}

Many of the studies evaluating the survival after cystectomy in the literature, suggest that ASA and ECOG scores predict the 90 days perioperative mortality in a substantive manner while $\mathrm{ACCl}$ predicts five-year OS more significantly. We have found out that the age of the patients has no sole effect on the oncologic results and that Framingham score and eGFR are much more precious and easily applicable parameters for the prediction of the PFS and OS and the Framingham score and MLR are for the prediction of the first 90 days mortality. Although ClavienDindo classification which categorizes the complications within the preoperative period, is one of the parameters that can not be applicable preoperatively, we are in the thought that it is particular importance in view of the postoperative follow up and treatment management due to the fact that is substantially predicts the first 90 days mortality, PFS and OS.

Nevertheless, in order to generalize our findings, we believe that long-term, prospective, multicentre, validated clinical studies are required, which include different risk classifications that predict perioperative - postoperative morbidity and mortality.

\section{Acknowledgements}

Publication: The results of the study were not published in full or in part in form of abstracts.
Contribution: There is not any contributors who may not be listed as authors.

Conflict of Interest: No conflict of interest was declared by the authors.

Financial Disclosure: The authors declared that this study received no financial support.

\section{Ethics}

Ethics Committee Approval: Due to the fact that this study is a retrospective, ethical committee approval was not obtained.

Informed Consent: Informed consent was obtained from the patients.

Peer-review: Externally peer-reviewed.

\section{Authorship Contributions}

Surgical and Medical Practices: H.B., Concept: H.B., Design: İ.S., Data Collection or Processing: I.S., Analysis or Interpretation: I.S., H.B., Literature Search: I.S., Writing: İ.S.

\section{References}

1. Jemal A, Bray F, Center MM, Ferlay J, Ward E, Forman D. Global cancer statistics. CA Cancer J Clin 2011;61:69-90.

2. Kuyumcuoğlu U, Özdedeli K. Radical Cystectomy in Elderly Patients: Predicting Complications and Mortality, Technical Aspects and Postoperative Care. Bulletin of Urooncology 2015;14:23-28.

3. Witjes AJ, Bruins HM, Cathomas R, et al. EAU guidelines on muscleinvasiveand metastatic bladdercancer: the 2019Update. https://www. uroweb/guideline/bladder-cancer-muscle-invasive-and-metastatic

4. Mayr R, May M, Martini T, et al. Predictive capacity of four comorbidity indices estimating perioperative mortality after radical cystectomy for urothelial carcinoma of the bladder. BJU Int 2012;110:E222-227.

5. Froehner $M$, Koch $R$, Heberling $U$, et al. Which comorbidity classification is best suited to identify patients at risk for 90-day and long-term non-bladder cancer mortality after radical cystectomy? World J Urol 2019.

6. Williams SB, Kamat AM, Chamie K, et al. Systematic review of comorbidity and competing risks assessments for bladder cancer patients. Eur Urol Oncol 2018;1:91-100.

7. St-Louis E, lqbal S, Feldman LS, et al. Using the age-adjusted Charlson comorbidity index to predict outcomes in emergency general surgery. J Trauma Acute Care Surg 2015;78:318-323.

8. Oken MM, Creech RH, Tormey DC, et al. Toxicity and response criteria of the Eastern Cooperative Oncology Group. Am J Clin Oncol 1982;5:649-655.

9. Saklad M. Grading of Patients for Surgical Procedures .Anesthesiology $1941 ; 2: 281-284$

10. Jahangiry L, Farhangi MA, Rezaei F. Framingham risk score for estimation of 10-years of cardiovascular diseases risk in patients with metabolic syndrome. J Health Popul Nutr 2017;36:36.

11. Joseph B, Zangbar B, Pandit $V$, et al. Emergency general surgery in the elderly: Too old or too frail? J Am Coll Surg 2016;222:805-813.

12. Boorjian SA, Kim SP, Tollefson MK, et al. Comparative performance of comorbidity indices for estimating perioperative and 5-year all cause mortality following radical cystectomy for bladder cancer. J Urol 2013;90:55-60.

13. Williams SB, Kamat AM, Chamie K, et al. Systematic review of comorbidity and competing risks assessments for bladder cancer patients. Eur Urol Oncol 2018;1:91-100.

14. Sathianathen NJ, Jarosek S, Lawrentschuk N, Bolton D, Konety BR. A Simplified Frailty Index to Predict Outcomes After Radical Cystectomy. Eur Urol Focus 2019;5:658-663. 
15. Froehner M, Koch R, Heberling U, Novotny V, Hübler M, Wirth MP. An easily applicable single condition-based mortality index for patients undergoing radical prostatectomy or radical cystectomy. Urol Oncol 2017;35:32.e17-32.e23.

16. Froehner $M$, Koch R, Hübler $M$, et al. Predicting 90-day and longterm mortality in octogenarians undergoing radical cystectomy. BMC Urol 2018; 18:91.

17. Lund L, Jacobsen J, Clark P, Borre M, Nørgaard M; Northern Danish Cancer Quality Assessment Group. Impact of comorbidity on survival of invasive bladder cancer patients, 1996-2007: a Danish populationbased cohort study. Urology 2010;75:393-398.

18. Fonteyne V, Ost P, Bellmunt I, et al. Curative treatment for muscle invasive bladder cancer in elderly patients: A systematic review. Eur Urol 2017;73:40-50.

19. Prout GR Jr, Wesley MN, Yancik R, Ries LA, Havlik RJ, Edwards BK. Age and comorbidity impact surgical therapy in older bladder carcinoma patients: a population-based study. Cancer 2005;104:1638-1647.

20. Mottet $N$ vdBR, Briers $E$, Cornford $P$, et al. European Association of Urology guidelines on prostate cancer: the 2019 Update. http:// uroweb.org/guideline/prostate-cancer/. ISBN 978-94-92671-04-2. EAU Guidelines Office, Arnhem, The Netherlands.

21. Sankar A, Johnson SR, Beattie WS, Tait G, Wijeysundera DN. Reliability of the American Society of Anesthesiologists physical status scale in clinical practice. Br J Anaesth 2014;113:424-432.

22. Ahmedov V, Kızılay F, Cüreklibatır I. Prognostic Significance of Body Mass Index and Other Tumor and Patient Characteristics in NonMetastatic Renal Cell Carcinoma. Urol J 2018;15:96-103.

23. Selvi I, Basar H. The Effect of Framingham Score on the Oncological Outcomes in Localized (T1-T2 Stage) Renal Cell Carcinoma Patients. Bull Urooncol 2019;18:59-66.
24. Matsumoto A, Nakagawa T, Kanatani A, et al. Preoperative chronic kidney disease is predictive of oncological outcome of radical cystectomy for bladder cancer. World J Urol 2018;36:249-256.

25. Grimes N, Hannan C, Tyson M, Thwaini A. The role of neutrophillymphocyte ratio as a prognostic indicator in patients undergoing nephrectomy for renal cell carcinoma. Can Urol Assoc I 2018;12:E345-348.

26. Yoshida T, Kinoshita H, Yoshida K, et al. Prognostic impact of perioperative lymphocyte-monocyte ratio in patients with bladder cancer undergoing radical cystectomy. Tumour Biol 2016;37:10067-10074.

27. Lucca I, Jichlinski P, Shariat SF, et al. The Neutrophil-to-lymphocyte Ratio as a Prognostic Factor for Patients with Urothelial Carcinoma of the Bladder Following Radical Cystectomy: Validation and Metaanalysis. Eur Urol Focus 2016;2:79-85.

28. Potretzke A, Hillman L, Wong K, et al. NLR is predictive of upstaging at the time of radical cystectomy for patients with urothelial carcinoma of the bladder. Urol Oncol 2014; 32:631-636.

29. Rajwa P, Życzkowski M, Paradysz A, Bujak K, Bryniarski P. Evaluation of the prognostic value of LMR, PLR, NLR, and dNLR in urothelial bladder cancer patients treated with radical cystectomy. Eur Rev Med Pharmacol Sci 2018;22:3027-3037.

30. Schulz GB, Grimm T, Buchner A, et al. Prognostic Value of the Preoperative Platelet-to-leukocyte Ratio for Oncologic Outcomes in Patients Undergoing Radical Cystectomy for Bladder Cancer. Clin Genitourin Cancer 2017;15:e915-e921. 